\title{
Nigrostriatal pathway degeneration in rats after intraperitoneal administration of proteasome inhibitor MG-132
}

\author{
Sławomir Wójcik, Jan Henryk Spodnik, Edyta Spodnik, Jerzy Dziewiątkowski, Janusz Moryś \\ Department of Anatomy and Neurobiology, Medical University of Gdańsk, Gdańsk, Poland
}

\begin{abstract}
The proteins' ubiquitination and their further degradation by proteasomes are crucial for cell cycle regulation, transcription and DNA replication, inflammatory response, and apoptosis. Proteasome inhibitors have recently become considered as a promising method in cancer and inflammatory disease therapy. In this study, utilizing the rat model, we try to establish the influence of proteasome inhibitor MG-132: (1) on the basis of spontaneous and evoked locomotor activity and (2) on the condition of nigrostriatal projections eight weeks after MG-132 intraperitoneal administration. We also discuss the current status of knowledge about intraperitoneal administration of MG-132, a laboratory method that is being used more and more. Our results revealed a lack of motor abnormalities, but significant loss (20\%) of substantia nigra pars compacta dopaminergic neurons after systemic MG-132 administration. This loss was accompanied by a corresponding decrease (8\%) of density of dopaminergic terminals in dorsolateral striatum. Moreover, evidence of very limited but ongoing fibre degeneration within the dorsal striatum suggests that MG-132 severely disturbed the nigrostriatal pathway.

In summary, intraperitoneal application of proteasome inhibitor MG-132, despite the encouraging results of experimental treatment and prevention of many pathological processes, should be used with caution because of the potential adverse effects on the structure of the central nervous system, especially elements of the nigrostriatal pathway.
\end{abstract}

Key words: Mg-132, proteasome inhibition, rat, striatum, substantia nigra, tyrosine hydroxylase.

\section{Introduction}

The ubiquitin-proteasome system is the main pathway of intracellular protein degradation within eukaryotic cells. The proteins' ubiquitination and their further degradation by proteasomes are crucial for cell cycle regulation, transcription and DNA replication, inflammatory response, and apoptosis. The proteasome inhibition leads to intracellular accumulation of misfolded or unfolded proteins and, in consequence, activation of numerous cell signalling pathways resulting in heatshock proteins expression increase, nuclear factor- $\mathrm{KB}$ (NFkB) and antiapoptotic cytokines decrease, mitochondrial protein expulsion into the cytoplasm, and caspase cascade activation $[10,48]$. Fast proliferating cells are more susceptible to ubiquitin-proteasome system disturbances. This is the reason why a new group of chemicals, called proteasome inhibitors, has recently been considered as a promising method in cancer and inflammatory disease therapy. 
MG-132 (Cbz-Leu-Leu-Leu-aldehyde, Z-LLL-H) is the most common and so far the most widely tested and best representative of both the peptide aldehydes and the entire group of proteasome inhibitors. It is a reversible inhibitor that gives a very quick response. MG-132 has a high degree of selective inhibition of proteasome chymotrypsin-like activity. A ten-fold higher concentration of the MG-132 inhibitor is needed to inhibit other enzymes (calpain and cathepsin), which indicates a specific inhibition of the proteasome. MG-132 properties, such as antiproliferative, anti-inflammatory, and induction of heat shock proteins, also make it the most widely used in the clinical impact of proteasome inhibition on the course of many processes at the cellular and tissue level, as well as having a link with studies of new proteasome inhibitors [15]. Literature reports indicate that under experimental conditions, intraperitoneal administration of MG-132 may play a beneficial role in the prevention and therapy of inflammatory diseases such as acute pancreatitis [9,24,50], inflammatory bowel disease [19], severe acute respiratory syndrome [28], and intestinal damage caused by ischaemia/reperfusion [1,21], and also partially prevents muscle atrophy associated with disuse [20]. However, data obtained on animal models are very controversial. Some authors [31,33] indicate that systematically administered proteasome inhibitors may penetrate the intact blood-brain barrier and cause pathological changes in the central nervous system (CNS), leading also to locomotor dysfunction and behavioural disturbances. It has also been reported that MG-132-induced hemiparkinsonism in the rat model mimics the daytime somnolence and sleep disturbance during the night observed in patients with the Parkinson's disease [26]. To date, there have been no reports describing the long-term effects of MG-132 on the CNS structures after its administration by intraperitoneal route. In this study utilizing the rat model we try to establish the influence of MG-132: (1) on the basis of spontaneous and evoked locomotor activity and (2) on the condition of nigrostriatal projections eight weeks after MG-132 intraperitoneal administration. We also discuss the current status of knowledge about intraperitoneal administration of MG-132, which is being used increasingly as a laboratory method.

\section{Material and methods}

Forty adult male Wistar rats (initial weight between 230 and $270 \mathrm{~g}$ ) were used in the study. They were kept under standard laboratory conditions: at $22^{\circ} \mathrm{C}$ temperature under an artificial light/dark cycle $(12 / 12$ h), with ad libitum access to tap water and standard laboratory food, three animals per large cage. Handling and care of the animals were in accordance with the National Institute of Health Guidelines for the Care and Use of Laboratory Animals. For all procedures prior approval from the local Bioethics Commission was obtained. All efforts were made to reduce the number of animals and to minimize their suffering.

In the first stage of the experiment, selection of the animals on the basis of their spontaneous locomotor activity was performed.

\section{A. Spontaneous activity - open field (automated)}

Measurements of spontaneous activity was performed in accordance with well-established protocols [12]. For assessing both exploratory and locomotor activity of the rats, the Video Track v.2.5 Automated Behavioural Analysis System (View Point Life Sciences Inc, Canada) was used. For open-field study, a quiet room with controlled ventilation and temperature was used. The animals were placed in the centre of white experimental boxes of adequate size - square arena $(50 \times 50 \mathrm{~cm})$ - surrounded by walls to prevent the animal from escaping. For ease of cleaning and to reduce behavioural response related to olfactory stimuli the experimental boxes were composed of plastic. At the same time, the activity of eight animals was video monitored. Initially, the rats were habituated to the apparatus during three sessions (10 minutes each) to reduce baseline activity. Then, spontaneous activity during light (with dimmed lighting) and dark phase (no lighting) - each one lasting 60 minutes, was recorded by the computer application Video Track VID 282 (View Point Life Sciences Inc, Canada). Image acquisition began 1 minute after the rats were placed in the box. Data were collected continuously and recorded each 30 seconds. The following parameters were evaluated:

- summary distance of exploratory movements [cm], - summary duration of exploratory movements [s], - summary distance of locomotor movements [cm], - summary duration of locomotor movements [s], - average velocity of locomotor movements [cm/s].

Due to the fact that differences in behaviour naturally occurring among animals divide them into those that exhibit greater motor activity in a novel environment (high responders - HR) and those that are less reactive (low responders - LR) [46], for further study only animals with a comparable activity 
as an average were selected, animals with high and low locomotor activity were excluded.

\section{B. Evoked motor activity - Rotarod test}

Selected animals $(n=30)$ underwent the Rotarod tests for balance and coordination assessed pre-operatively at five speeds of rotation: 10, 12, 14, 16 and $18 \mathrm{rpm}$, to establish a baseline performance. For this test, the apparatus comprised of a rotating drum (Ugo Basile, Italy) was used. Four rats were placed on the revolving drum, one per testing station, and once they were balanced the stepper motor was started. The speed of the motor was increased to $10 \mathrm{rpm}$ for 10 seconds and then progressively to 12,14 , and $16 \mathrm{rpm}$ for 10 seconds each. The highest speed, $18 \mathrm{rpm}$, was maintained for 300 seconds until the 5-minute test period elapsed. To evaluate motor function the measurement of latency (time in seconds) to fall from the rotating drum was taken. The timer began automatically when the motor was switched on and stopped when the animal fell down to the floor of the apparatus. Each animal had three consecutive trials, the longest time on the drum being used for analysis. The animals were exposed to the Rotarod each day for 3 days to adapt the rats to the apparatus. The rats were then intraperitoneally injected with $10 \%$ DMSO or MG-132 solution according to the protocol described below and tested once per week during eight weeks of observation on the Rotarod for periods of 300 seconds. The means of the test results were used for statistical analysis.

\section{MG-132 administration}

The animals were randomly divided into three groups: control $(n=10)$, which received an intraperitoneal injection of $10 \%$ DMSO solution and two groups of rats that received a reversible proteasome inhibitor - MG-132 (lot \# 3570428, Boston Biochem Inc., USA) dissolved in 10\% DMSO. Protocol applications for both groups were based on the protocol proposed by McNaught et al. [33] for the application of other compounds from the group of proteasome inhibitors. Briefly, the rats received intraperitoneal injections of MG-132 every other day (total of 7 doses) for a period of two weeks. The first group of MG-132 rats $(n=10)$ received a higher dose (10 $\mu \mathrm{g} / \mathrm{g}$ of body weight) of the inhibitor, while the second group $(n=10)$ received a lower dose $(0.5 \mu \mathrm{g} / \mathrm{g}$ of body weight). Both doses administered to the animals were based on the those previously reported in the literature. The higher dose was used in the studies of $[9,24,50]$, and the lower dose in the studies of $[1,21]$.

\section{Tissue collection and preparation}

Eight weeks after first MG-132 administration the rats were deeply anaesthetized intraperitoneally with lethal doses of Nembutal ( $80 \mathrm{mg} / \mathrm{kg}$ of body weight), and then they were transcardially perfused with $0.9 \%$ saline containing 10,000 units of Heparin, followed by $4 \%$ solution of paraformaldehyde in $0.1 \mathrm{M}$ phosphate buffer $\left(\mathrm{pH} 7.4 ; 4^{\circ} \mathrm{C}\right)$. Directly after perfusion, the brains were taken out of the skulls, postfixed in 4\% paraformaldehyde for 3-4 hours, and then cryoprotected initially in $10 \%$ (overnight at $4^{\circ} \mathrm{C}$ ) and subsequently in $30 \%$ sucrose phosphate buffer solution $\left(\mathrm{pH} 7.4 ; 4^{\circ} \mathrm{C}\right)$. After the brains soaked in the solution (1-3 days), each left hemisphere was marked by slight damage of the neocortex, and then the brains were cut on a cryostat (Microm HM 525, Thermo Scientific) into $40 \mu \mathrm{m}$ thick coronal sections. For further studies, sections were collected and preserved in a cryoprotective solution containing glycerol.

\section{Immunohistochemistry}

To evaluate the possible changes made by MG-132 administration in the population of dopaminergic neurons in substantia nigra pars compacta (SNc), immunohistochemical staining with primary antibody against tyrosine hydroxylase (TH), a well-established marker of dopaminergic neurons, was performed. To evaluate all populations of SNc neurons double staining with anti-TH antibody and Neuro Trace 500/525 green fluorescent Nissl Stain (lot \# 649182, Invitrogen, Molecular probes, USA) was performed.

Briefly, free-floating sections were blocked in a 3\% normal goat serum (NGS) and 0.3\% Triton X-100 in 0.01 M PBS ( $\mathrm{pH}$ 7.2) for 1 hour at room temperature. Next, they were incubated with primary antibodies: mouse monoclonal anti-TH (Chemicon, USA; $1: 500$ ) in $0.01 \mathrm{M}$ PBS ( $\mathrm{pH} 7.2$ ) containing NGS and $0.1 \%$ Triton $\mathrm{X}-100$ at $4^{\circ} \mathrm{C}$. After 48 hours the sections were washed with PBS and incubated ( 2 hours, room temperature) with secondary goat anti-mouse antibody coupled with Cy3 (Jackson, USA; 1 : 800). Finally, they were washed with PBS, mounted onto gelatine-coated slides, air-dried and coverslipped with Keiser Gelatin (Merck, Germany). Omission of the primary antibodies during control experiments resulted in a lack of signal.

For the unequivocal qualitative detection of both gross and fine scale neuronal degeneration, staining with Fluoro-Jade ${ }^{\circledR}$ B (Chemicon; Millipore) was used. 
The manufacturer's "Protocol for using Fluoro-Jade ${ }^{\circledR}$ B" was applied for the single and double (with anti-TH antibodies) sections staining.

\section{Qualitative analysis}

The low-magnification images and initial analysis of immunostained sections were performed on an MVX10 MacroView research Macro Zoom Fluorescence Microscope (Olympus, Japan) equipped with a XC50 digital camera (Olympus, Japan). The highmagnification images and co-localization study were performed using a confocal laser scanning microscopy (CLSM) system (Radiance 2100, Bio-Rad UK) mounted on a microscope (Eclipse 600, Nikon, Japan). The CLSM images were obtained with $40 x$ and $60 x$ oil immersion objective lenses of N.A. $=1.3$ and 1.4, respectively. The optimal iris diameter was used for each magnification. The CLSM images were analysed with LaserSharp 2000 and LaserPix v. 2.0 software (both Bio-Rad, UK).

\section{Quantitative analysis}

The CellSense Dimension 1.5 (Olympus, Japan) image analysis system was employed to assess the density of TH-ir positive cells in the substantia nigra pars compacta. The area of interest was outlined and computer-aided estimation was used to calculate the amount of TH-ir cells in the SNc. The number of TH-ir positive cells counted for both SNc on a separate slice was estimated individually for each animal. Representative sections of SNc were selected (between Bregma -4.80 and Bregma -6.04) [35]. All cells visible on the outlined area of a given section were analysed. Quantification of intensity of TH-staining within the striatum was performed according to the following method. The micrographs obtained with the aid of an image analysis system, CellSense Dimension 1.5 (Olympus, Japan), were converted into greyscale images (256 shades of grey) for measurement of TH fluorescence intensity. For morphometric analysis, greyscale intensity (Gl; range 0-255: black $=0$, white $=255)$ of $\mathrm{TH}$-ir and background area were simultaneously measured using ImageJ 1.46R analysis software (NIH, USA) on a personal computer. The intensity of proportional TH-stained areas within dorsomedial, dorsolateral, and ventral striatum were measured through the rostrocaudal axis of the striatum. To compare the intensity of the sections the data are presented as proportional values of staging intensity (measured from 0 to
255) of a given area versus intensity of TH-ir-negative background. Calculation of the density of SNc dopaminergic and non-dopaminergic neurons, as well as total number of SNc neurons, was also performed by analysis with LaserPix v. 2.0 (Bio-Rad, UK) CSLM images obtained with a $40 X$ lens and zoom set to 1.9 from double stained sections. The size of the testing area was $165 \times 165 \mu \mathrm{m}$. At least ten areas from each animal were studied. The first test area within SNc was chosen randomly and the remaining ones were selected by systematic random sampling.

Measurements were carried out in 5 animals from each studied group (at least 3 sections/animal).

\section{Statistical analysis}

Data are expressed as mean \pm standard errors of mean (SEM). To test the results for statistical significance, the following were used: (a) for data obtained from open field test - a nonparametric test SteelDwass: Pairwise Comparisons for One-Way Layout Design; (b) for data obtained from Rotarod test $-2 \times 2$ contingency table and Fisher test; (c) for TH-ir profiles distribution comparison $-\chi^{2}$ test for normality; (d) for the rest of the parameters - statistics were performed with original data via unpaired $t$-test. Differences were considered significant when $p<0.05$.

\section{Results}

In the group of rats after intraperitoneal administration of MG-132 at a dose of $10 \mu \mathrm{g} / \mathrm{g}$ of body weight, the mortality was $40 \%$ during 24 hours after the first dose, and then an additional $40 \%$ during the next 24 hours. Therefore, we did not pursue further experiments in this trial group.

In the group of rats that were given an intraperitoneal injection of MG-132 at a dose $0.5 \mu \mathrm{g} / \mathrm{g}$ of body weight and in the control group, mortality was not observed. There were no significant differences between the control group and MG-132 group in the changes of body weight during eight weeks of observation.

\section{Intraperitoneal injection of MG-132 does not affect exploratory activities and locomotor activity adequate to the circadian rhythm}

A week after the last administration of $0.5 \mu \mathrm{g} / \mathrm{g}$ MG-132 dose, the first evaluation of spontaneous activity in an open field test was performed (Fig. 1). 
We found that in the control group and in the group treated with MG-132 adequate to the circadian rhythm, locomotor activity was maintained. There were no significant differences between the groups in the studied parameters of locomotor activity. During the light phase of open field test, the average summary distance of locomotor movements in the control group was $93.2 \pm 26.4 \mathrm{~cm}$ while in the MG-132 group it was $148.9 \pm 90.6 \mathrm{~cm}$. In both groups, the value of this parameter was significantly higher ( $p<0.05$ ) during the dark phase of the open field test: $779.7 \pm 200.0 \mathrm{~cm}$ and $774.7 \pm 82.3 \mathrm{~cm}$, respectively. The summary duration of locomotor movements during the light phase in the control group was $12.4 \pm 2.3$ seconds while in the MG-132 group it was $17.3 \pm 11.1$ seconds, and during the dark phase it was $91.6 \pm 23.6$ and $88.8 \pm 8.9$ seconds, respectively. The average velocity of locomotor movements did not differ significantly in both groups between the light and dark phases. In the control group, the values of this parameter were (light phase) $8.3 \pm$ $0.2 \mathrm{~cm} / \mathrm{s}$ and (dark phase) $8.2 \pm 0.2 \mathrm{~cm} / \mathrm{s}$, while in the MG-132 group they were $8.5 \pm 0.1 \mathrm{~cm} / \mathrm{s}$ and $8.8 \pm$ $0.1 \mathrm{~cm} / \mathrm{s}$, respectively.

There were also no significant differences between the control group and MG-132 group in the studied pa- rameters of exploratory movement. During the light phase of the open field test, the average summary distance of exploratory movements in the control group was $183.3 \pm 26.4 \mathrm{~cm}$ while in the MG-132 group it was $494.8 \pm 192.3 \mathrm{~cm}$. The value of this parameter was higher during the dark phase of the open field test: $843.4 \pm 217.2 \mathrm{~cm}$ and $1300.2 \pm 249.7 \mathrm{~cm}$, respectively. The summary duration of exploratory movements during the light phase in the control group was $48.8 \pm 11.1$ seconds while in the MG-132 group it was $129.3 \pm 21.3$ seconds, and during the dark phase it was $200.2 \pm 51.6$ and $319.3 \pm 70.1$ seconds, respectively.

Very similar results were obtained during a second open field test performed eight weeks after the start of the experiment (after the first dose, MG-132 intraperitoneal administration).

\section{Intraperitoneal injection of MG-132 does not influence motor activity and coordination}

Evaluation of forced motor activity and coordination of animals was performed at the end of each eight consecutive weeks of observation. Only in the third week were there any observed differences between the control group and MG-132 group. Whereas all control rats could remain on the rotating
Control - light phase

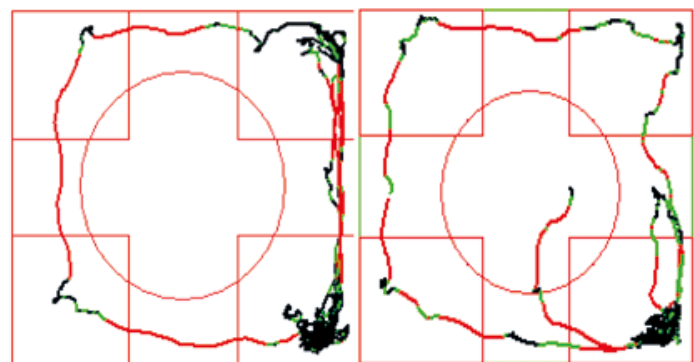

Control - dark phase
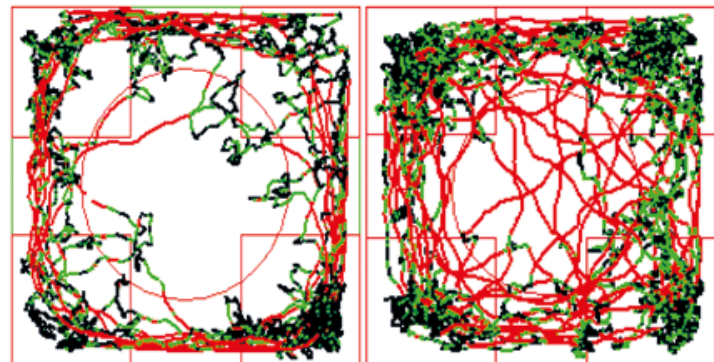

MG-132 - light phase

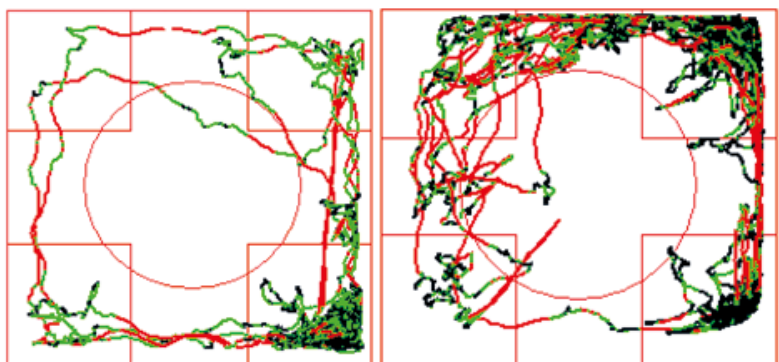

MG-132 - dark phase
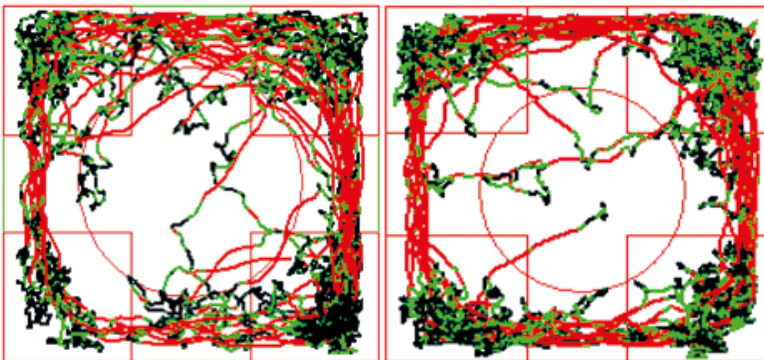

Fig. 1. Representative graphs of spontaneous activity recorded during light (with dimmed lighting) and dark phase (no lighting) of the experiment in control and MG-132 i.p. administered rats. Red colour represents locomotor movement, green colour represents exploratory movement. 
rod for close to the full 300 seconds duration of the test, $40 \%$ of the rats from the MG-132 group fell off the rod sooner, but it was not statistically significant. During the remaining Rotarod tests the average results obtained in both groups were almost equal.

\section{Intraperitoneal injection of MG-132 causes quantitative and qualitative changes in the population of rats substantia nigra pars compacta TH-ir neurons}

The rats were sacrificed eight weeks after the beginning of the experiment - first intraperitoneal administration of MG-132 in DMSO or 10\% DMSO (for control). Dual immunohistochemical staining followed by image analysis and cell counting revealed that the number of neuronal profiles within the SNc of the rats administered with MG-132 was significantly decreased by $17.1 \%(p<0.01)$ as compared to the control animals (Fig. 2). A similar significant loss - of $20.1 \%(p<0.01)$, of TH-positive neurons within the SNc of the rats administered with MG-132 as compared to control animals was also observed. There were no significant changes between the number of non-TH-ir nigral neurons between the studied groups of rats (Fig. 2). These results indicate that dopaminergic neurons within the SNc undergo degeneration, not just the loss of possibility for $\mathrm{TH}$ expression. It also shows that populations of SNc non-dopaminergic neurons were not as susceptible to MG-132 as dopaminergic ones were.

Within the population of SNC TH-ir neurons, in addition to quantitative, qualitative changes were also observed. Image analysis of SNc TH-ir cell profiles from controls $(n=952)$ and MG-132 administered rats $(n=839)$ revealed that distribution of those cells profiles differed significantly $\left(\chi^{2}=115.116\right.$, $p<0.0001$ ) between the studied groups. As is shown on the comparative distribution graph (Fig. 2), within the SNc of MG-132 administered rats there was a marked decrease of large (bigger than $200 \mu \mathrm{m}^{2}$ ) TH-ir neurons.

\section{Intraperitoneal injection of MG-132 induces $\mathrm{TH}$-ir elements loss in rat striatum}

In the rat striatum, the immunohistochemical study revealed the presence of $\mathrm{TH}$-ir fibres located predominantly within the matrix (Fig. 3A). Their diverse morphology closely corresponded to that reported by Gerfen and colleagues [16]. The most frequently observed were relatively thin (0.1-0.4 $\mu \mathrm{m})$, smooth fibres with small varicosities (0.25-0.65 $\mu \mathrm{m})$. These fibres were present within the matrix. They correspond to Gerfen's type A fibres, being axon terminal dopaminergic cells from the dorsal tier of SNc (dorsal A9 cell group). Much less frequently, thicker fibres $(0.2-0.65 \mu \mathrm{m})$ with larger varicosities $(0.4-1.0 \mu \mathrm{m})$ were present. They were predominantly located in the proximity of striosomes. Their morphology and localization corresponded to type B fibres, being axon terminals dopaminergic cells from the ventral tier of SNc (ventral A9 cell group) and the ventral tier of substantia nigra reticulata (displaced A9 cell group).

The intensity of TH-staining in the striatum of the rats after intraperitoneal administration of MG-132 compared to the density of $\mathrm{TH}$-ir fibres in the control animals (Fig. 4) revealed that in the dorsomedial part of the striatum and ventral striatum no significant differences were observed (values of calculated intensity factors were 1.57 vs. 1.63 and 1.53 vs. 1.55 , respectively). A significant, $8 \%$ decrease $(p<0.05)$ of TH-ir in the dorsolateral part of the striatum (1.28 in the MG-132 group vs. 1.39 in the control group, Fig. 4) was observed. A decrease in TH staining due to the loss of dopaminergic fibres was similar along the rostrocaudal axis of the striatum. This localization of TH-ir loss may indicate that while limbic and associative areas of the striatum are relatively resistant, the sensorimotor areas are more susceptible to damage caused by MG-132 administration. FluoroJade B staining, detecting both gross and fine-scale neuronal degeneration, showed the presence of degenerating fibres in the dorsal striatum (Fig. 3B). Those fibres were present in both striatal compartments, within the matrix and striosomes, but only on brain sections from animals after MG-132 administration. Features of degenerating fibres morphology were very similar to those present on $\mathrm{TH}$-containing axons innervating the striatum (Fig. 3). In addition to the fibres corresponding to type $A$ and type $B$, occasionally (1-2 per section), a third category of fibres in the matrix were observed. They were thicker $(0.45-1.05 \mu \mathrm{m})$, and possessed vesicular, much larger varicosities (1.5-2.9 $\mu \mathrm{m})$. Their morphology and localization corresponded to type $C$ fibres, being axon terminals of non-dopaminergic cells from SN. Double-labelling Fluoro-Jade B/tyrosine hydro- 
A

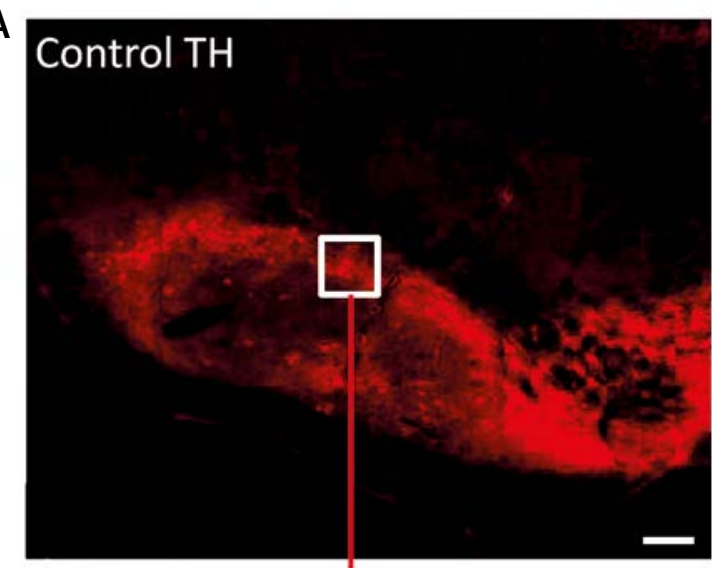

B
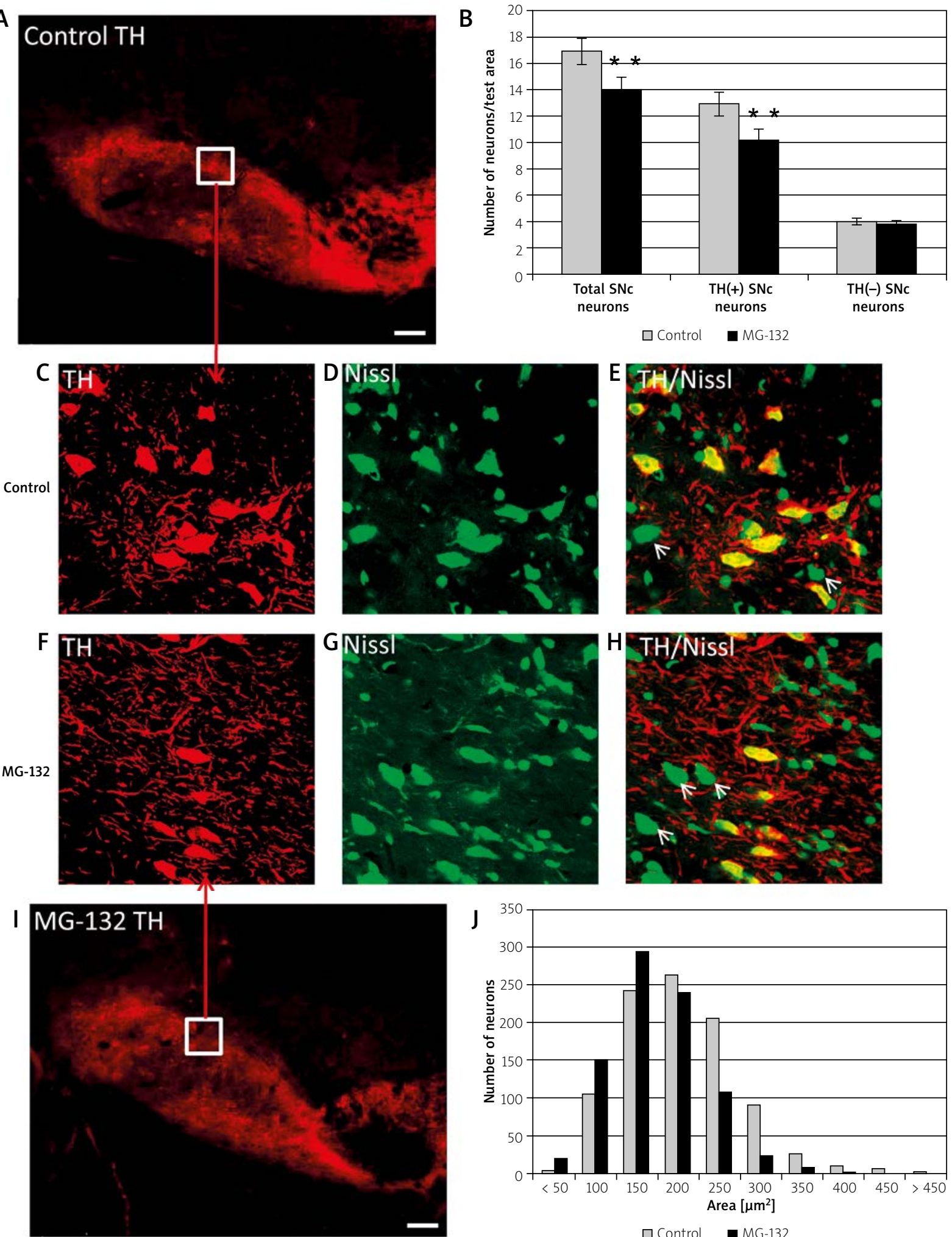

Fig. 2. Loss of TH-ir neurons in SNc in response to MG-132 administration. Normal density of TH-ir A, C (red), $\mathrm{E}$ (yellow) and non TH-ir E (green, marked with arrows) neurons in SNc in control animals. Loss of TH-ir F, I (red), $\mathbf{H}$ (yellow) and non TH-ir $\mathbf{H}$ (green, marked with arrows) neurons in SNc in MG-132 animals. Graph B shows a significant loss of all neurons and $\mathrm{TH}(+)$ neurons, but not $\mathrm{TH}(-)$ in SNc of rats administered with MG-132. Graph J shows distribution of $\mathrm{TH}(+)$ profiles according to their size within $\mathrm{SNc}$ of studied groups. Scale bar $200 \mu \mathrm{m}$. 


\section{Control}

A

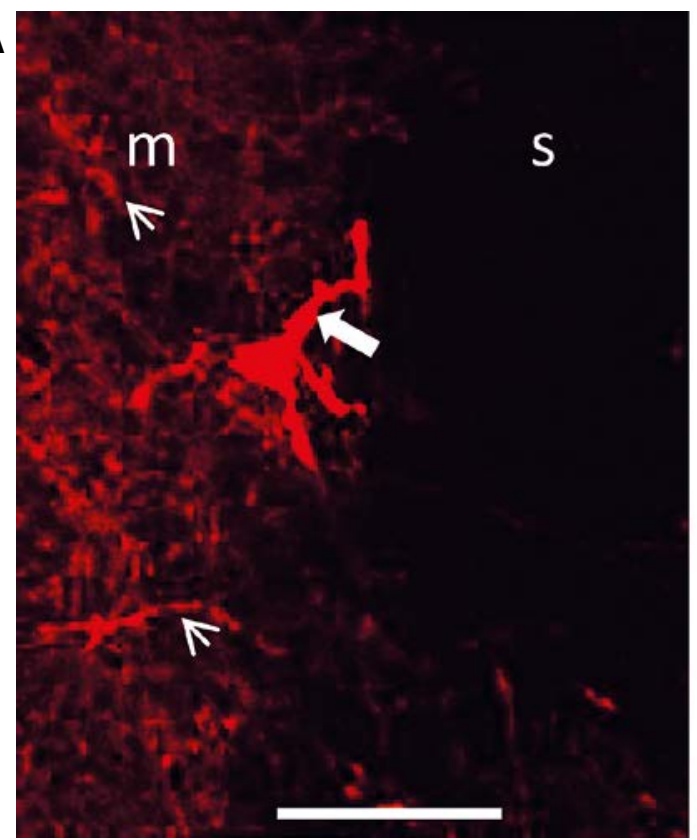

MG-132 TH

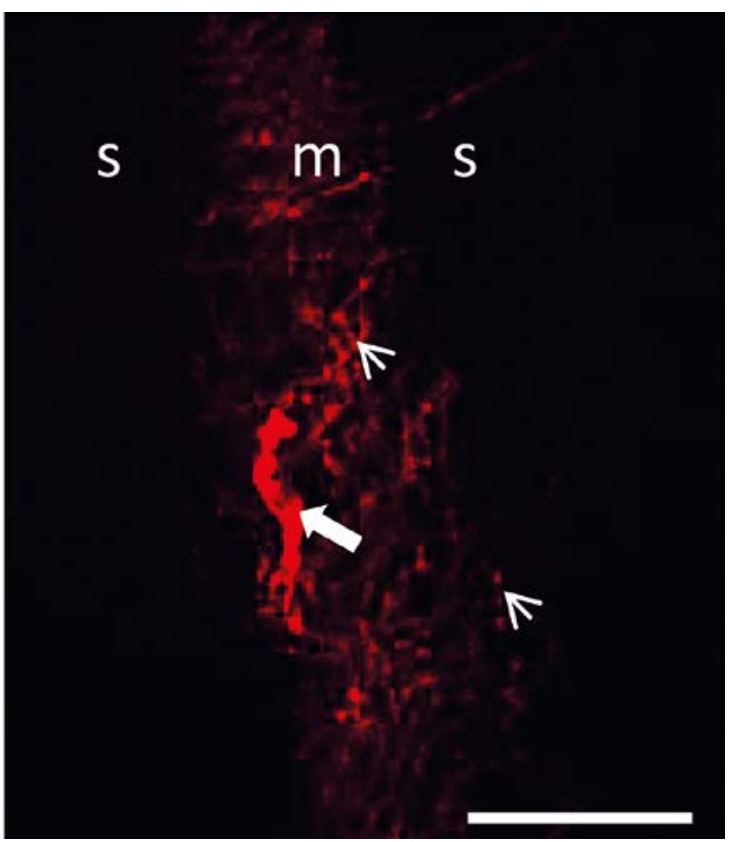

B

MG-132 FluoroJade B
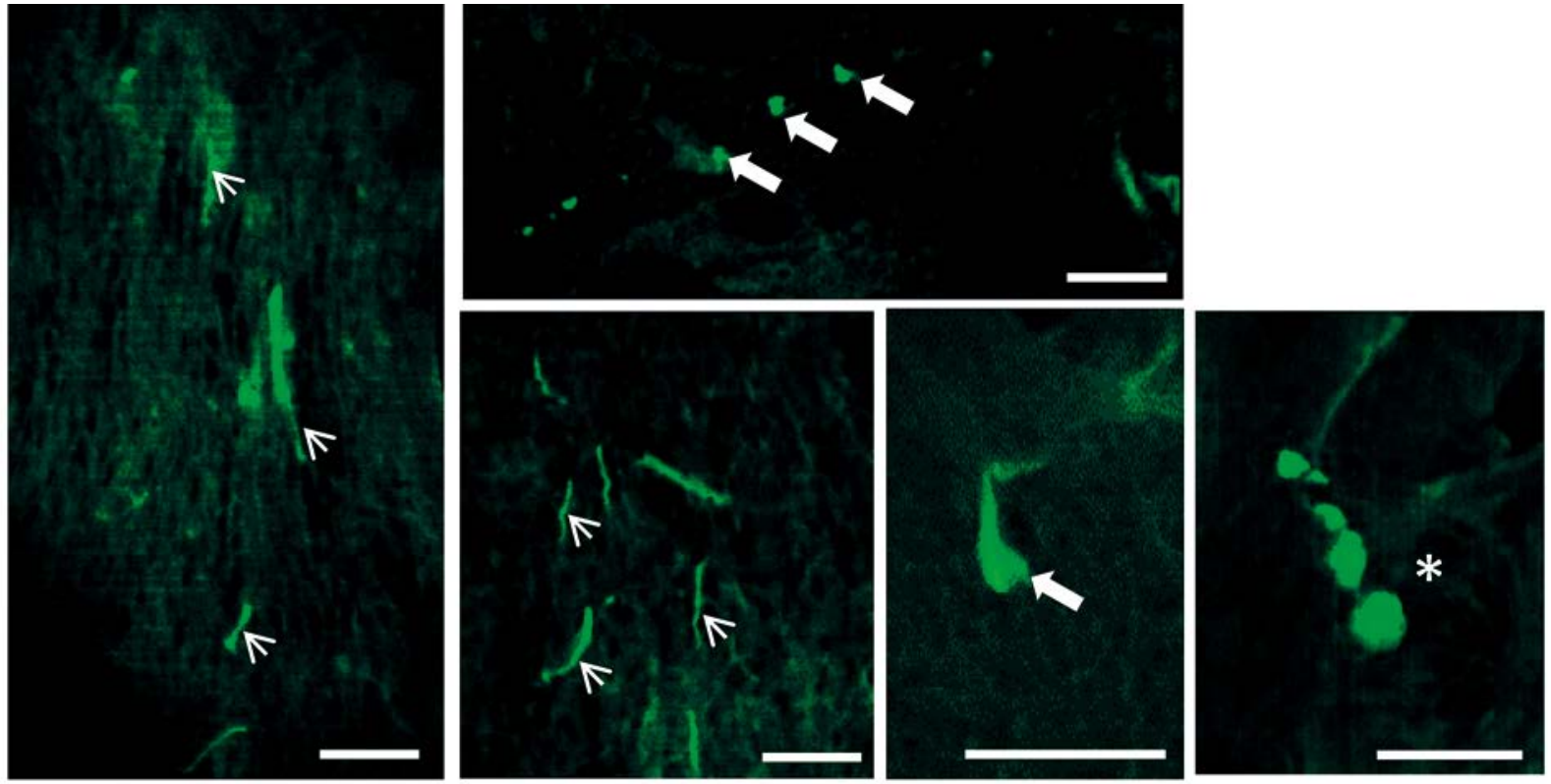

Fig. 3. Morphology of the fibres within the striatum. A) Distribution and morphology of TH-ir type A fibres (small arrows) and type B fibres (large arrows) within the striatum of studied groups. B) Morphology of degenerating fibres revealed by Fluoro-Jade B staining. Type A fibres (small arrows), type B fibres (large arrows), type C fibres (asterisk) within the striatum of MG-132 rats - detailed description in the text. (s) - striosome; $(\mathrm{m})$ - matrix. Scale bar $10 \mu \mathrm{m}$. 
A
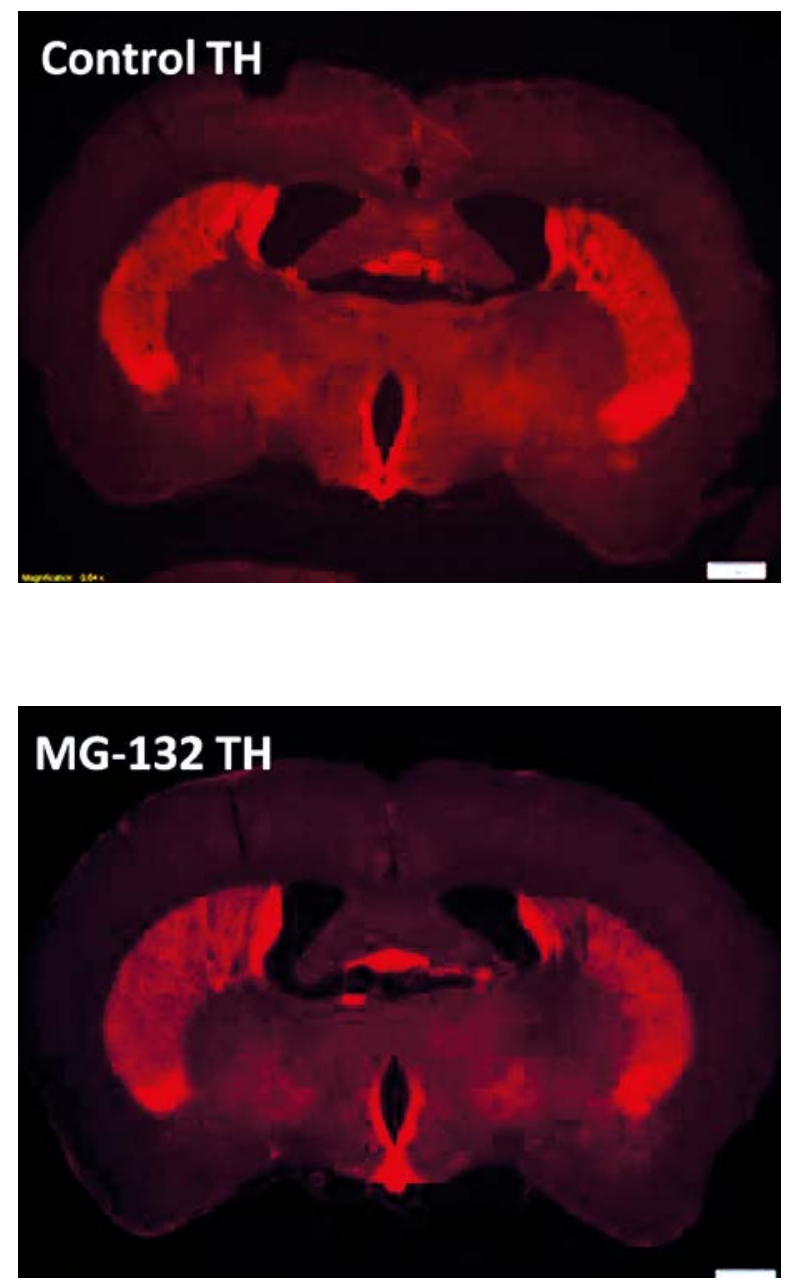

B

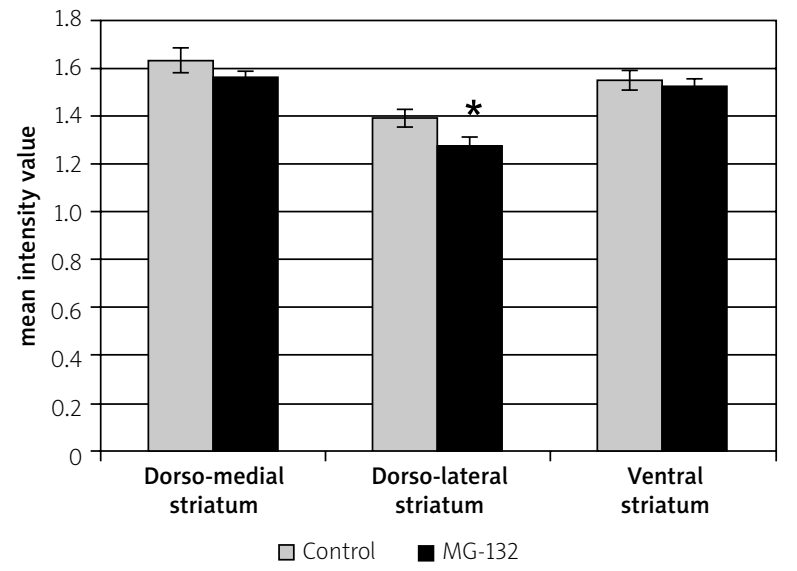

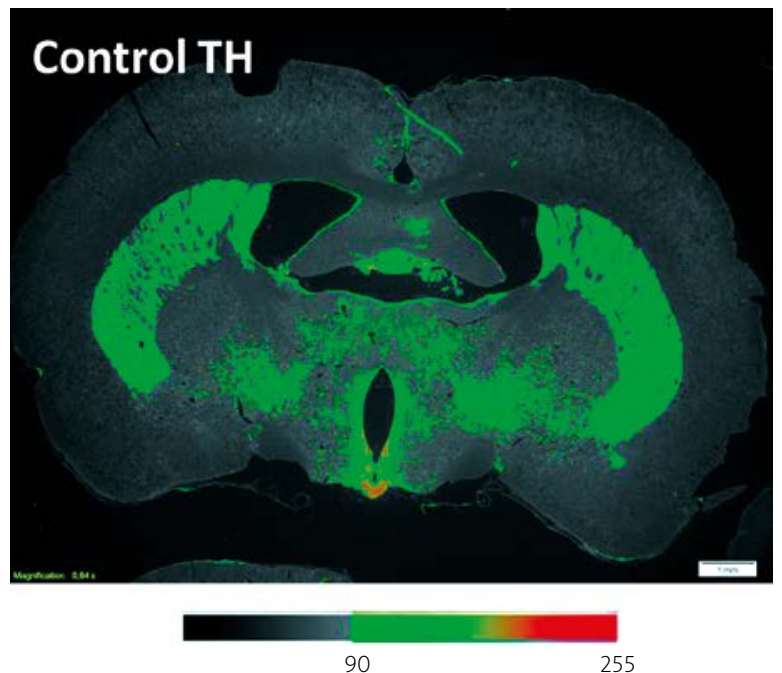

90

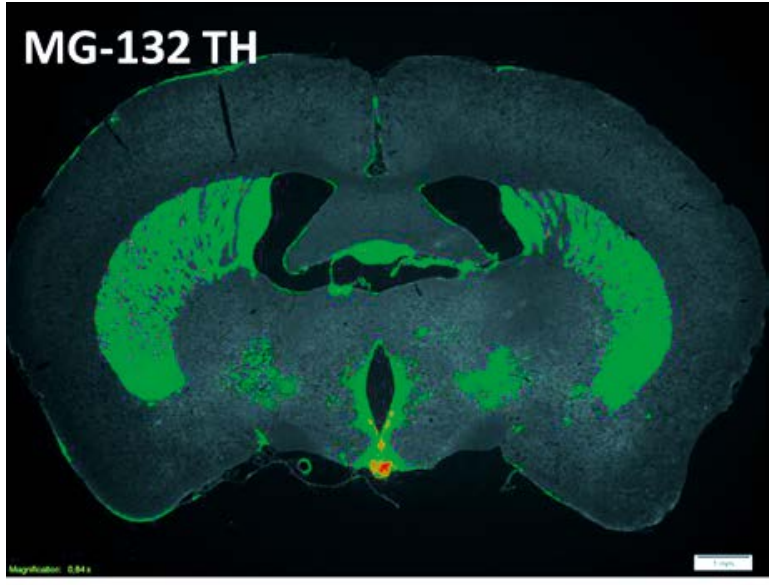

90

255

Fig. 4. Loss of $\mathrm{TH}$-ir fibres in the striatum in response to MG-132 administration. A) The upper panel shows normal density of $\mathrm{TH}$-ir fibres (red) and pseudo colour intensity (scale included) in the striatum of control animals. The lower panel shows a small loss of $\mathrm{TH}$-ir fibres (red) and pseudo colour intensity (scale included) in the striatum of MG-132 animals. B) The graph shows a significant loss of $\mathrm{TH}(+)$ fibres in dorsolateral striatum but not in dorsomedial striatum and ventral striatum of rats administered with MG-132. Scale bar $1 \mathrm{~mm}$. 
A

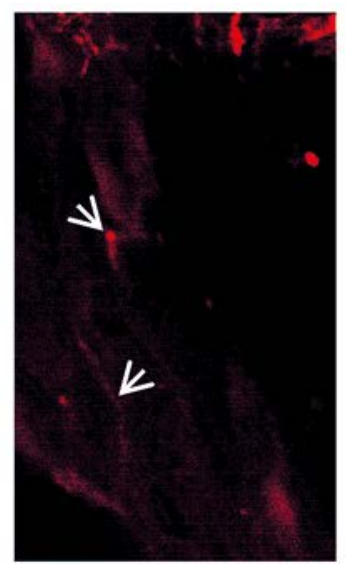

B

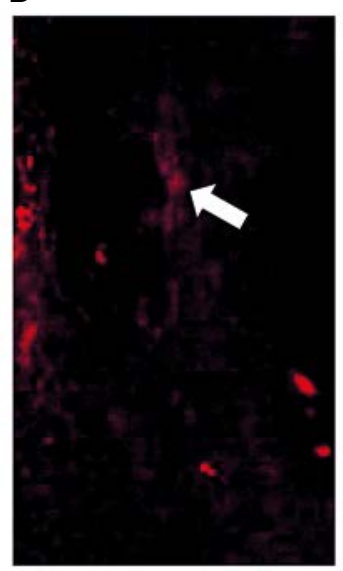

C

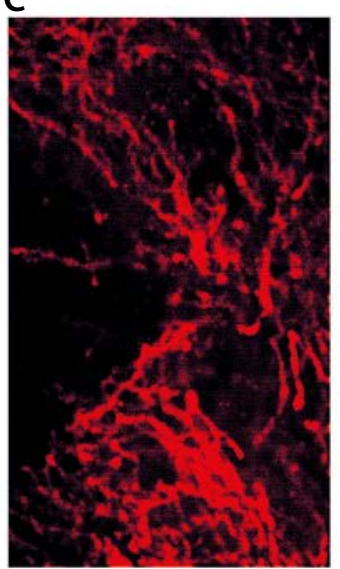

FluoroJade B
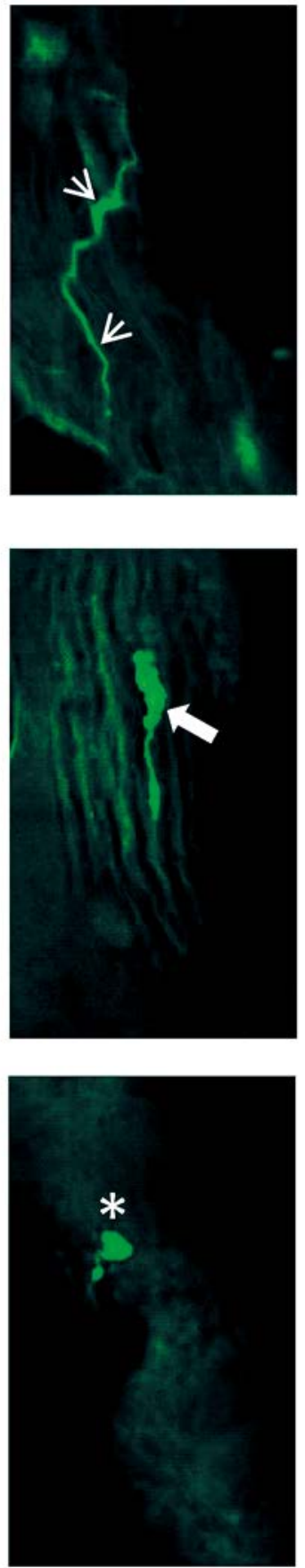

Merge TH/FluoroJade B
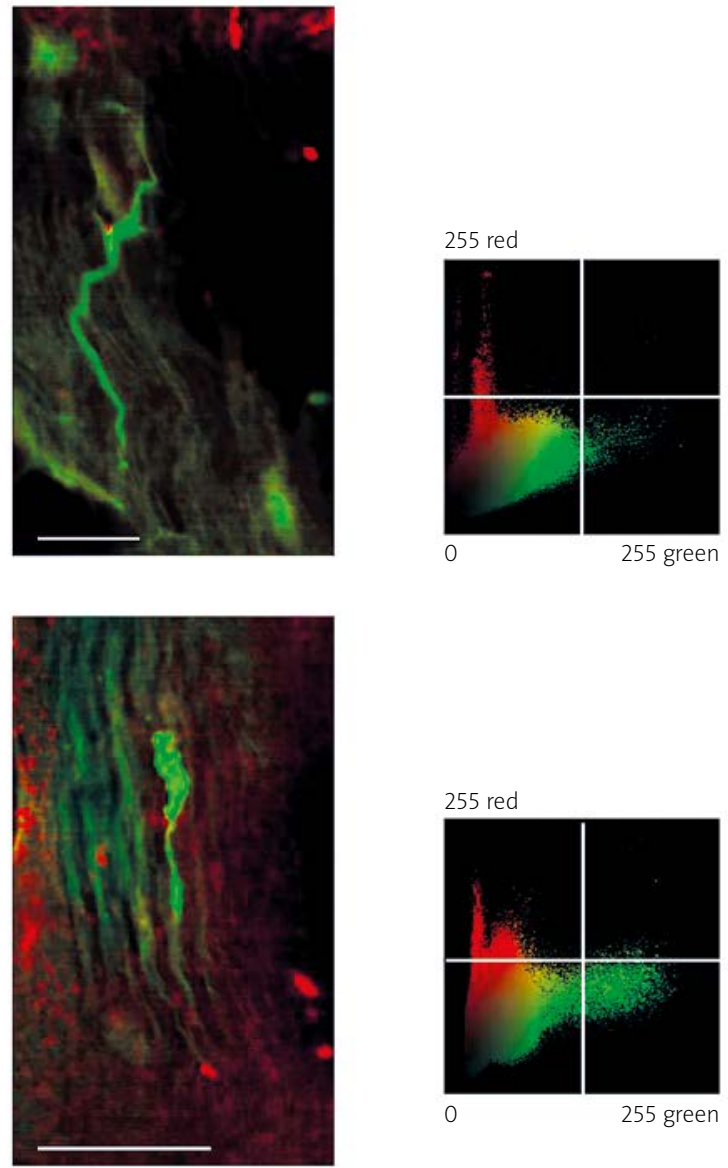

0

255 green

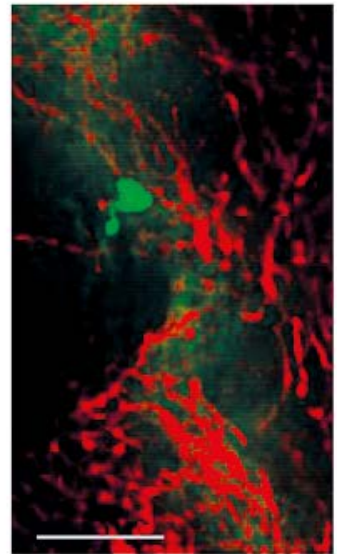

255 red

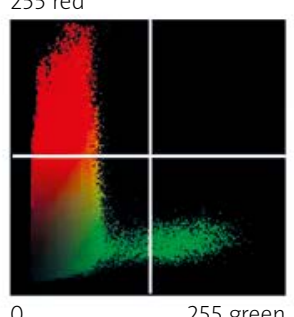

Fig. 5. Fluoro-Jade B/tyrosine hydroxylase $(\mathrm{TH})$ double-labelling in the dorsal striatum of MG-132 administered rats. Degenerating fibres, Fluoro-Jade B positive, showed various morphologies - corresponding to type A of normal TH-fibres (A), fibres with large varicosities, type $\mathrm{B}$ of normal TH-fibres (B) up to the type $\mathrm{C}$ fibres with a collection of vesicular varicosities (C). Very low intensity of TH immunoreactivity (indicated by arrows) were detected in degenerating fibres with type $A$ and $B$ morphology (A, B). At the type C fibres (C) Fluoro-Jade B and TH positive elements comprised two separate populations. It is also visible on the 2D colocalization plots (with intensities of red colour plotted against intensities of green colour) corresponding to the confocal images (A-C). Scale bar $10 \mu \mathrm{m}$. 
xylase $(\mathrm{TH})$ revealed that in the dorsal striatum of MG-132 administered rats' degenerating fibres, very low intensity of $\mathrm{TH}$ immunoreactivity (Fig. 5) was detected in degenerating fibres with type $A$ and $B$ morphology. In the type $C$ fibres, Fluoro-Jade $B$ and $\mathrm{TH}$ positive elements comprised two separate populations. This has also been confirmed by co-localization studies.

\section{Discussion}

After Alzheimer's disease, Parkinson's disease is the second most common neurodegenerative disorder. It affects more than $1 \%$ of the world population aged 65 years. Clinical symptoms of Parkinson's disease (PD) include non-motor signs: olfactory deficits, sleep disturbances, depression, psychosis, and cognitive impairment as well as more spectacular motor signs: muscular rigidity, bradykinesia, resting tremor, and postural instability. The latter result from the destruction of the substantia nigra dopaminergic neurons projecting to the striatum, and loss of dopamine transmission in the motor loops of the basal ganglia. To date pathogenesis of PD has remained largely elusive and the general consensus is that PD is a multifactorial disease: a combination of genetic inheritance, aging and some unknown environmental factors. A growing number of reports provide evidence that inhibition of the ubiquitin-proteasome system (UPS) plays a role in the pathogenesis of PD [49]. Confirmation of UPS impairment in the brains of PD patients given by findings of accumulation of UPS components and its substrates in Lewy bodies, reduced proteasome activity in substantia nigra, and the presence of PD in patients with mutations in gene encoding for several UPS-related key molecules.

A great deal of possible information about the pathogenesis of PD has been obtained due to in vivo results from various animal models of this disease $[3,5,40,44]$. Among them, degenerative models with MPTP and rotenone are the most commonly used. However, recently it has been postulated that direct administration into elements of the nigrostriatal system $[14,25,45]$ or systemic administration of proteasome inhibitors may serve as a new animal model of PD. The basis of this theory was the results of animal studies indicating that systemically administered (intraperitoneally) proteasome inhibitors like PSI and epoxomicin may, after absorption into the bloodstream, penetrate the intact blood-brain barrier and cause lesions at the substantia nigra, locus coeruleus, and the Meynert nucleus, and in consequence, lead to an impairment of the motor function $[32,33,38]$. This allowed the researches to hypothesize that the use of proteasome inhibitors could be used to obtain a new animal model of Parkinson's disease. It should be noted that not all groups of researchers were able to obtain the same results $[6,23]$.

However, there are two previous reports describing the influence of MG-132 on the nigrostriatal pathway $[41,49]$. According to our knowledge, the current report is the first to estimate the influence of systemic administration of MG-132. The results we have shown in this report reveal a significant loss of SNc dopaminergic neurons in rats six weeks after the end of systemic, intraperitoneal MG-132 administration. This loss was accompanied by a corresponding decrease in density of dopaminergic fibres within the dorsolateral striatum. Moreover, evidence of very limited but ongoing $\mathrm{TH}$-ir fibre degeneration within the dorsal striatum suggests that MG-132 severely disturbed the nigrostriatal pathway. Our observation that the number of SNc TH-ir cells in the rats intraperitoneally injected with small doses of MG-132 decreased by $20.1 \%$ corresponds to the results shown by Xie et al. [49]. They found that injection of $0.4 \mathrm{mg}$ of MG-132 into the medial forebrain bundle in mice decreased the number of nigral $\mathrm{TH}$-ir cells by $24.5 \%$. The degree of SNc dopaminergic neuron loss observed by us is smaller than that produced by direct injection of $0.4 \mu \mathrm{g}$ MG-132 in $4 \mu \mathrm{l}$ 1\% DMSO into the substantia nigra reported by Sun et al., which was approximately $40 \%$ [41]. Those discrepancies suggest that the different administration routes of the same proteasome inhibitor may lead to different consequences. This was also recently shown for another proteasome inhibitor - lactacystin. A unilateral, intranigral injection of lactacystin at doses of $0.5,1,2.5$, and $5 \mu \mathrm{g} / 2 \mu \mathrm{l}$ produced after 7 days, distinct decreases in the concentrations of dopamine (DA) and its metabolites (DOPAC, 3-MT, HVA) in the ipsilateral striatum. Such alterations were not observed in the striatal DA content and catabolism either 7, 14, or 21 days after a unilateral, intrastriatal high-dose lactacystin injection (5 and $10 \mu \mathrm{g} / 2 \mu \mathrm{l}$ ) [25].

The results of a previous study evaluating the influence of systemic administration of proteasome inhibitors on behavioural and neuropathological features of Parkinson's disease are ambiguous. In 
some experimental conditions, mainly after subcutaneous administration of PSI, a loss of SN dopaminergic neurons was observed and varied between $40 \%$ [38] and 50\% [51], and even exceeded 50\% [33]. Four other groups of researchers, despite the fact that they tried faithfully to follow the experimental protocol proposed by McNaught et al., failed to replicate the results described earlier $[6,22,23,30]$. Our results support the theory of McNaught et al.

The striatum is responsible for integrating incoming information from all areas of the cerebral cortex and creating motor patterns based on past knowledge (procedural memory) and on the recent environment and desires [11]. The striosome/matrix cytoarchitectonic structures of the striatum play a crucial role in its functional organization. Afferent connections present in the matrix, especially in the lateral part of the dorsal striatum, are related to sensomotor areas of the cerebral cortex. Matrix located more medially and striosomes have predominant associative- and limbic-related connections, respectively [11]. Similarly to data from previous successful demonstration of systemic proteasome inhibitors' effect on damage of nigrostriatal connections $[32,33,38,51]$, we observed a loss of dopaminergic terminals within the striatum. Previous reports indicated a decrease in dopaminergic innervations of the striatum by 30 to $40 \%$. We observed that after intraperitoneal MG-132 administration the decrease of $\mathrm{TH}$ staining, due to the loss of dopaminergic fibres, was similar along the rostrocaudal axis of the striatum, but significant by $8 \%$ only in the lateral part of the dorsal striatum. The changes observed by us in the distribution of the striatal TH-ir fibres correspond to previous data obtained under other experimental conditions [39]. After MPTP administration in monkeys the smallest loss of striatal TH-ir fibres named as "mild stage" was observed in the dorsolateral striatum. It suggests that the sensorimotor areas of the striatum in the rat are more susceptible to damage, including damage caused by MG-132 administration, while limbic and associative areas are relatively resistant.

Under our experimental conditions, the loss of nigral TH-ir neurons in the SNc and dopaminergic terminals in the dorsolateral striatum was not accompanied by the presence of motor abnormalities, as shown by a series of behavioural tests of spontaneous and forced locomotion. The lack of significant differences in spontaneous and forced loco- motor activity observed by us appears to result from a relatively high amount (80\%) of dopaminergic neurons ( $\mathrm{TH}-\mathrm{ir}$ ), which remained in substantia nigra pars compacta and $92 \%$ of dopaminergic terminals in striatum. According to earlier reports, during Parkinson's disease at the onset of symptoms, putamenal dopamine is depleted by approximately $\approx 80 \%$, and approximately $\approx 60 \%$ of SNc dopaminergic neurons have already been lost [13]. The results of other studies showed that motor signs of PD manifest after the loss of approximately $50 \%$ of nigral neurons and $80 \%$ of striatal dopamine $[4,47]$. Two postmortem studies using more rigorous quantitative methods, such as using a tessellation method [17] and dissector-based neuromorphometry [27], found that about $30 \%$ of nigral neuronal loss appeared sufficient to cause motor symptoms in PD [2]. However, recent research suggests that the first symptoms of Parkinson's disease may manifest after just a $20 \%$ decrease in the number of dopaminergic neurons SNc, which has been shown in animal models where a reduction of about 14 to $23 \%$ of nigral neuron count or $14 \%$ to $37 \%$ of striatal dopamine was sufficient to induce mild parkinsonism [43].

\section{Systemic administration of proteasome inhibition}

In rats with $50 \%$ loss of SNc dopaminergic neurons after systemic PSI administration no bradykinesia or akinesia was observed, but they did exhibit a reduction by 30 to $40 \%$ of exploratory behaviour. The $20 \%$ loss of dopaminergic cells in SNc and $8 \%$ loss of dopaminergic terminals in striatum, as observed by us, may not be sufficient to produce motor symptoms of PD.

Intraperitoneal administration of proteasome inhibitor Mg-132 is a method known for almost ten years; however, it is not applied universally. It is worth noting that attempts were made to use MG-132 for the treatment and prevention of a wide range of pathologies in animal models. The influence of intraperitoneal injection MG-132 has been studied in two aspects: (1) its immediate influence, evaluated in hours after administration and (2) its delayed influence, evaluated in days and weeks (up to 12 ) after administration.

Injected intraperitoneally, a single dose of MG132, varying from 0.5 to $10 \mathrm{mg} / \mathrm{kg}$ b.w., was able to successfully act on the elements of the digestive 
system. Observations made after intraperitoneal MG-132 administration have become the basis for a theory of the beneficial role of proteasome inhibitors not only in prevention, but also in the therapy of acute pancreatitis $[9,24,50]$. This beneficial effect is dependent on the induction of the expression of cell-protective heat shock proteins (Hsp72), modulation of DNA-binding of nuclear NFKB, suppression of the elevation of pancreatic myeloperoxidase activity, tumor necrosis factor $\alpha$ (TNF- $\alpha$ ), and intracellular adhesion of molecule- 1 and serum amylase. Intraperitoneal administration of MG-132 also decreases inflammatory response, by lowering the increase in interleukin 1 (IL-1), TNF- $\alpha$, and IL-10 levels in the caecal ligation and puncture model of sepsis [36]. Studies by Alexandrova et al. showed that intraperitoneal MG-132 treatment activates the liver antioxidant enzyme system e.g. superoxide dismutase, catalase and glutathione peroxidase [1]. They postulated that MG-132 might play a preventive role in the development of cell and tissue injury. It was recently confirmed [21] in the rat model that intraperitoneal administration of MG-132 has a significant effect in protection against liver injury induced by intestinal ischaemia/reperfusion, most probably due to modulation of $N F \kappa B$ and Aryl hydrocarbon receptor pathways. A single intraperitoneal dose of MG-132 appears also to act effectively on the structures belonging to systems other than the gastrointestinal. According to Chen et al., intraperitoneal administration of MG-132 shows a protective effect on lung injury in rats by significantly decreasing pulmonary myeloperoxidase activity [9]. However, the most comprehensive study of immediate response to intraperitoneal MG-132 administration was published by Holecek et al. [18]. They reported a significant increase in protein synthesis not only in the digestive system (liver), but also in the kidney and skeletal muscle. In addition, they observed a significant decrease in proteasome-dependent proteolysis in skeletal muscle and the increase in protein turnover associated with a significant increase in proteasome-dependent proteolysis in the liver and kidney.

Authors more often indicate a possible systemic toxic effect and even an increase in the mortality risk in the reports where there were late effects of MG-132 and the effects of multiple doses of the MG-132 studied [29,37]. A dose of $10 \mathrm{mg} / \mathrm{kg}$ of body weight (cumulative dose of above $30 \mathrm{mg} / \mathrm{kg}$ ) provided intraperitoneally seems to lead to high mortality, although rats can survive a cumulative dose of $105 \mathrm{mg} / \mathrm{kg}$ [7] delivered in 14 doses during seven days subcutaneously. After prolonged MG-132 administration, its delayed influence has been reported on more numerous structures from many systems. It has been reported that [19] in experimental inflammatory bowel disease MG-132 in vivo reduced T cell-mediated intestinal inflammation but significantly suppressed cell migration and epithelial cell proliferation. MG-132 also has an influence on the cardiovascular system $[8,29,34]$. In a low dose $(0.1 \mathrm{mg} / \mathrm{kg})$ MG-132 administered intraperitoneally once per day even for 2 or 8 weeks may effectively prevent cardiac remodelling and dysfunction in pressure-overloaded hearts without marked drug toxicity [29]. The effects of intraperitoneal injection of MG-132 was also studied on urinary [37], musculoskeletal [20,42], and respiratory systems [28]. The study revealed that MG-132 is not an adequate treatment to prevent endotoxin-induced diaphragmatic dysfunction $[20,42]$ but partially prevents muscle atrophy associated with disuse [20]. MG-132 administered in relatively large doses (4 mg/kg, repeated seven times) did not ameliorate tubulointerstitial fibrosis in rat unilateral ureteral obstruction [37]. Positive effects of MG-132, namely attenuation pneumonitis and cytokine gene expression in vivo by reducing coronavirus were observed [28].

In summary, intraperitoneal application of proteasome inhibitor MG-132, despite the encouraging results of the experimental treatment and prevention of many pathological processes, should be used with caution because of the potential adverse effects on the structure of the central nervous system, especially elements of the nigrostriatal pathway.

\section{Acknowledgement}

Supported by Grant Funds from National Scientific Committee - N N401 005735.

\section{References}

1. Alexandrova A, Petrov L, Georgieva A, Kirkova M, Kukan M. Effects of proteasome inhibitor, MG132, on proteasome activity and oxidative status of rat liver. Cell Biochem Funct 2008; 26 : 392-398.

2. Beach TG, Adler CH, Sue LI, Peirce JB, Bachalakuri J, Dalsing-Hernandez JE, Lue LF, Caviness JN, Connor DJ, Sabbagh MN, Walker DG. Reduced striatal tyrosine hydroxylase in incidental Lewy body disease. Acta Neuropathol 2008; 115: 445-451. 
3. Betarbet R, Sherer TB, Greenamyre JT. Animal models of Parkinson's disease. Bioessays 2002; 24: 308-318.

4. Bezard E, Dovero S, Prunier C, Ravenscroft P, Chalon S, Guilloteau D, Crossman AR, Bioulac B, Brotchie JM, Gross CE. Relationship between the appearance of symptoms and the level of nigrostriatal degeneration in a progressive 1-methyl-4-phenyl-1,2,3,6-tetrahydropyridine-lesioned macaque model of Parkinson's disease. J Neurosci 2001; 21: 6853-6861.

5. Blesa J, Phani S, Jackson-Lewis V, Przedborski S. Classic and new animal models of Parkinson's disease. J Biomed Biotechnol 2012; 2012: 845618.

6. Bove J, Zhou C, Jackson-Lewis V, Taylor J, Chu Y, Rideout HJ, Wu DC, Kordower JH, Petrucelli L, Przedborski S. Proteasome inhibition and Parkinson's disease modeling. Ann Neurol 2006; 60: 260-264.

7. Caron AZ, Haroun S, Leblanc E, Trensz F, Guindi C, Amrani A, Grenier $\mathrm{G}$. The proteasome inhibitor MG132 reduces immobilization-induced skeletal muscle atrophy in mice. BMC Musculoskelet Disord 2011; 12: 185.

8. Chen B, Ma Y, Meng R, Xiong Z, Zhang C, Chen G, Zhang A, Dong Y. MG132, a proteasome inhibitor, attenuates pressure-overload-induced cardiac hypertrophy in rats by modulation of mitogen-activated protein kinase signals. Acta Biochim Biophys Sin (Shanghai) 2010; 42: 253-258.

9. Chen X, Li SL, WU T, Liu JD. Proteasome inhibitor ameliorates severe acute pancreatitis and associated lung injury of rats. World I Gastroenterol 2008; 14: 3249-3253.

10. Ciechanover A. Intracellular protein degradation: from a vague idea thru the lysosome and the ubiquitin-proteasome system and onto human diseases and drug targeting. Biochim Biophys Acta 2012; 1824: 3-13.

11. Crittenden JR, Graybiel AM. Basal Ganglia disorders associated with imbalances in the striatal striosome and matrix compartments. Front Neuroanat 2011; 5: 59.

12. Curzon P, Zhang M, Radek RJ, Fox GB. The Behavioral Assessment of Sensorimotor Processes in the Mouse: Acoustic Startle, Sensory Gating, Locomotor Activity, Rotarod, and Beam Walking. 2009.

13. Dauer W, Przedborski S. Parkinson's disease: mechanisms and models. Neuron 2003; 39: 889-909.

14. Duty S, Jenner P. Animal models of Parkinson's disease: a source of novel treatments and clues to the cause of the disease. $\mathrm{Br}$ J Pharmacol 2011; 164: 1357-1391.

15. Fritsch Fredin M, Vidal A, Utkovic H, Gotlind YY, Willen R, Jansson L, Hultgren Hornquist E, Melgar S. The application and relevance of ex vivo culture systems for assessment of IBD treatment in murine models of colitis. Pharmacol Res 2008; 58: 222-231.

16. Gerfen CR, Herkenham M, Thibault J. The neostriatal mosaic: II. Patch- and matrix-directed mesostriatal dopaminergic and non-dopaminergic systems. J Neurosci 1987; 7: 3915-3934.

17. Greffard S, Verny M, Bonnet AM, Beinis JY, Gallinari C, Meaume S, Piette F, Hauw JJ, Duyckaerts C. Motor score of the Unified Parkinson Disease Rating Scale as a good predictor of Lewy body-associated neuronal loss in the substantia nigra. Arch Neurol 2006; 63: 584-588.

18. Holecek M, Muthny T, Kovarik M, Sispera L. Proteasome inhibitor MG-132 enhances whole-body protein turnover in rat. Biochem Biophys Res Commun 2006; 345: 38-42.
19. Inoue S, Nakase H, Matsuura M, Mikami S, Ueno S, Uza N, Chiba T. The effect of proteasome inhibitor MG132 on experimental inflammatory bowel disease. Clin Exp Immunol 2009; 156: 172-182.

20. Jamart C, Raymackers JM, Li An G, Deldicque L, Francaux M. Prevention of muscle disuse atrophy by MG132 proteasome inhibitor. Muscle Nerve 2011; 43: 708-716.

21. Jing $H$, Shen G, Wang G, Zhang F, Li Y, Luo F, Yao J, Tian XF. MG132 Alleviates liver injury induced by intestinal ischemia/ reperfusion in rats: involvement of the AhR and NFkappaB pathways. J Surg Res 2012; 176: 63-73.

22. Kadoguchi N, Kimoto H, Yano R, Kato H, Araki T. Failure of acute administration with proteasome inhibitor to provide a model of Parkinson's disease in mice. Metab Brain Dis 2008; 23: 147-154.

23. Kordower JH, Kanaan NM, Chu Y, Suresh Babu R, Stansell J, 3rd, Terpstra BT, Sortwell CE, Steece-Collier K, Collier TJ. Failure of proteasome inhibitor administration to provide a model of Parkinson's disease in rats and monkeys. Ann Neurol 2006; 60: 264-268.

24. Letoha T, Feher LZ, Pecze L, Somlai C, Varga I, Kaszaki J, Toth G, Vizler C, Tiszlavicz L, Takacs T. Therapeutic proteasome inhibition in experimental acute pancreatitis. World J Gastroenterol 2007; 13: 4452-4457.

25. Lorenc-Koci E, Lenda T, Antkiewicz-Michaluk L, Wardas J, Domin $\mathrm{H}$, Smialowska M, Konieczny J. Different effects of intranigral and intrastriatal administration of the proteasome inhibitor lactacystin on typical neurochemical and histological markers of Parkinson's disease in rats. Neurochem Int 2011; 58: 839-849.

26. Lu CY, Yi PL, Tsai CH, Cheng CH, Chang HH, Hsiao YT, Chang FC. TNF-NF-kappaB signaling mediates excessive somnolence in hemiparkinsonian rats. Behav Brain Res 2010; 208: 484-496.

27. Ma SY, Roytta M, Rinne JO, Collan Y, Rinne UK. Correlation between neuromorphometry in the substantia nigra and clinical features in Parkinson's disease using disector counts. J Neurol Sci 1997; 151: 83-87.

28. Ma XZ, Bartczak A, Zhang J, Khattar R, Chen L, Liu MF, Edwards A, Levy G, McGilvray ID. Proteasome inhibition in vivo promotes survival in a lethal murine model of severe acute respiratory syndrome. J Virol 2010; 84: 12419-12428.

29. Ma Y, Chen B, Liu D, Yang Y, Xiong Z, Zeng J, Dong Y. MG132 treatment attenuates cardiac remodeling and dysfunction following aortic banding in rats via the NF-kappaB/TGFbeta1 pathway. Biochem Pharmacol 2011; 81: 1228-1236.

30. Manning-Bog AB, Reaney SH, Chou VP, Johnston LC, McCormack AL, Johnston J, Langston JW, Di Monte DA. Lack of nigrostriatal pathology in a rat model of proteasome inhibition. Ann Neurol 2006; 60: 256-260.

31. Matsui H, Ito H, Taniguchi Y, Inoue H, Takeda S, Takahashi R. Proteasome inhibition in medaka brain induces the features of Parkinson's disease. J Neurochem 2010; 115: 178-187.

32. MCNaught KS, Olanow CW. Proteasome inhibitor-induced model of Parkinson's disease. Ann Neurol 2006; 60: 243-247.

33. McNaught KS, Perl DP, Brownell AL, Olanow CW. Systemic exposure to proteasome inhibitors causes a progressive model of Parkinson's disease. Ann Neurol 2004; 56: 149-162. 
34. Meiners S, Hocher B, Weller A, Laule M, Stangl V, Guenther C, Godes M, Mrozikiewicz A, Baumann G, Stangl K. Downregulation of matrix metalloproteinases and collagens and suppression of cardiac fibrosis by inhibition of the proteasome. Hypertension 2004; 44: 471-477.

35. Paxinos G, Watson C. The rat brain in stereotaxic coordinates. $4^{\text {th }}$ ed. Academic Press, San Diego, London 1998.

36. Safranek R, Ishibashi N, Oka Y, Ozasa H, Shirouzu K, Holecek M. Modulation of inflammatory response in sepsis by proteasome inhibition. Int J Exp Pathol 2006; 87: 369-372.

37. Sakairi T, Hiromura K, Takahashi S, Hamatani H, Takeuchi S, Tomioka M, Maeshima A, Kuroiwa T, Nojima Y. Effects of proteasome inhibitors on rat renal fibrosis in vitro and in vivo. Nephrology (Carlton) 2011; 16: 76-86.

38. Schapira AH, Cleeter MW, Muddle JR, Workman JM, Cooper JM, King RH. Proteasomal inhibition causes loss of nigral tyrosine hydroxylase neurons. Ann Neurol 2006; 60: 253-255.

39. Song DD, Haber SN. Striatal responses to partial dopaminergic lesion: evidence for compensatory sprouting. J Neurosci 2000; 20: 5102-5114

40. Stoica G, Lungu G, Bjorklund NL, Taglialatela G, Zhang X, Chiu V, Hill HH, Schenk JO, Murray I. Potential role of alpha-synuclein in neurodegeneration: studies in a rat animal model. J Neurochem 2012; 122: 812-822.

41. Sun F, Anantharam V, Zhang D, Latchoumycandane C, Kanthasamy A, Kanthasamy AG. Proteasome inhibitor MG-132 induces dopaminergic degeneration in cell culture and animal models. Neurotoxicology 2006; 27: 807-815.

42. Supinski GS, Vanags J, Callahan LA. Effect of proteasome inhibitors on endotoxin-induced diaphragm dysfunction. Am J Physiol Lung Cell Mol Physiol 2009; 296: L994-L1001.

43. Tabbal SD, Tian L, Karimi M, Brown CA, Loftin SK, Perlmutter JS. Low nigrostriatal reserve for motor parkinsonism in nonhuman primates. Exp Neurol 2012; 237: 355-362.

44. Valastro B, Dekundy A, Krogh M, Lundblad M, James P, Danysz W, Quack G, Cenci MA. Proteomic analysis of striatal proteins in the rat model of L-DOPA-induced dyskinesia. J Neurochem 2007; 102: 1395-1409.

45. Vernon AC, Johansson SM, Modo MM. Non-invasive evaluation of nigrostriatal neuropathology in a proteasome inhibitor rodent model of Parkinson's disease. BMC Neurosci 2010; 11: 1

46. White DA, Kalinichev M, Holtzman SG. Locomotor response to novelty as a predictor of reactivity to aversive stimuli in the rat. Brain Res 2007; 1149: 141-148.

47. Wolters E. Non-motor extranigral signs and symptoms in Parkinson's disease. Parkinsonism Relat Disord 2009; 15 Suppl 3 : S6-12.

48. Wu WK, Cho CH, Lee CW, Wu K, Fan D, Yu J, Sung JJ. Proteasome inhibition: a new therapeutic strategy to cancer treatment. Cancer Lett 2010; 293: 15-22.

49. Xie W, Li X, Li C, Zhu W, Jankovic J, Le W. Proteasome inhibition modeling nigral neuron degeneration in Parkinson's disease. J Neurochem 2010; 115: 188-199.

50. Xu CF, Xia SJ. Effect of MG-132 upon the expression of intercellular adhesion molecule-1 in cerulein-induced acute pancreatitis in mice. Zhonghua Yi Xue Za Zhi 2010; 90: 853-856.
51. Zeng BY, Bukhatwa S, Hikima A, Rose S, Jenner P. Reproducible nigral cell loss after systemic proteasomal inhibitor administration to rats. Ann Neurol 2006; 60: 248-252. 